\title{
CRISTALES ORGÁNICOS LASER: ANTRACENO
}

\section{ORGANIC CRYSTALS LASER: ANTHRACENE}

\author{
Aníbal Valera Palacios ${ }^{1}$, Miguel Melchor Vivanco², Miguel Ángel Mosquera Molina ${ }^{3}$
}

\begin{abstract}
RESUMEN
En esta contribución se presenta la técnica de elaboración de cristales orgánicos por el método de sublimación aplicado al caso particular de Antraceno. Se presentan asimismo los últimos resultados experimentales obtenidos en nuestro Laboratorio con los monocristales de Antraceno: A) Luminiscencia a temperatura ambiente. Los cristales fueron sometidos a irradiancia UV-Vis frontal, obteniéndose la transmitancia frontal y la fluorescencia de canto (wave guide emisión). Como resultado de estas medidas se identifica un cuasigap de $3.11 \mathrm{eV}$ (398 nm) y bandas de emisión a niveles vibronicos en 3.08, 2.92, 2.79 y $2.61 \mathrm{eV}$. B) Conversión Fotovoltaica. Se construyó la unión fotovoltaica Antraceno / oxido de estaño (Ant / TO), determinándose experimentalmente la eficiencia espectral quántica del sistema, así como el modelo teórico energético correspondiente.
\end{abstract}

Palabras clave.- Antraceno, Eficiencia cuántica, Conversión fotovoltaica.

\begin{abstract}
In this contribution are shown organic crystals obtained by the sublimation method and the latest experimental results obtained with the Anthracene monocrystals: A) Luminescence at room temperature. The Anthracene crystals were subjected to frontal UV-Vis irradiance, obtaining the frontal transmittance and the edge Fluorescence (wave guide emission) at room temperature. As a result of this measurements we identified a "quasigap" of $3.11 \mathrm{eV}(398 \mathrm{~nm})$ and emission bands in 3.08, 2.92, 2.79 and $2.61 \mathrm{eV}$. B) Photovoltaic conversion. We constructed photovoltaic junctions Anthracene / Tin oxide (Ant / TO). We determined experimentally the quantum spectral efficiency of the system and the corresponding theoretical band energy diagram.
\end{abstract}

Keywords. - Anthracene, Quantum efficiency, Photovoltaic conversion.

\section{INTRODUCCIÓN}

La motivación principal del estudio intensivo desarrollado en los últimos años a nivel mundial en el crecimiento y propiedades físicas de materiales orgánicos, surge del gran potencial de aplicaciones opto electrónicas factibles de realizar con estos materiales; entre las que cabe destacar: Celdas solares [1] [2], Dispositivos LED (OLEDs) [3], Transistores de película fina (OTFTs) [4].
Guías ópticas y acopladores ópticos [5], Fotodetectores [6] y Láseres [7]. Los descubrimientos realizados independientemente por Kepler [8] y LeBlanc [9] referentes a la movilidad de electrones y huecos en monocristales de antraceno, hace cerca de 40 años, constituyeron un paso decisivo en la introducción de los materiales orgánicos a esferas técnicas reservadas casi exclusivamente a los semiconductores inorgánicos, la electrónica y fotónica.

${ }^{1}$ Dr. Docente investigador de la Facultad de Ciencias de la Universidad Nacional de Ingeniería, ${ }^{2}$ Alumno del Laboratorio de Óptica de la Facultad de Ciencias de la Universidad Nacional de Ingeniería, ${ }^{3}$ Alumno del Laboratorio de Óptica de la Facultad de Ciencias de la Universidad Nacional de Ingeniería. 
No obstante, el gran número de aplicaciones posibles de realizar con los materiales orgánicos, su performance y optimización depende aun del entendimiento físico de estos materiales y de sus propiedades opto eléctricas, motivo por el cual son tema de estudio de gran número de centros de Investigación a nivel mundial (por ejemplo: III Phys. Inst. derUni. Stuttgart / Germany). Recientemente, cristales poliacénicos como Antraceno han atraído nuevamente el interés científico [10], debido a sus novedosas propiedades y distintas aplicaciones.

Desde el punto de vista conceptual, muchos grupos de investigación se centran actualmente en estudiar principalmente, la física del transporte en los materiales orgánicos (excitones, cargas, energía) [11] y sus uniones, para lo cual se requiere acumular mayor información a partir de mediciones experimentales.

En este sentido, este trabajo presenta una contribución referente a las propiedades de Monocristales de antraceno obtenidos en nuestros laboratorios.

\section{DATOS REFERENCIALES}

En esta sección se describen las características básicas del cristal Antraceno que serán tomadas como fundamento para explicar los resultados experimentales que se presentan en este trabajo.

\section{ANTRACENO}

\section{Estructura}

En la Fig. 1 se presenta esquemáticamente la molécula de Antraceno $\mathrm{C}_{14} \mathrm{H}_{10}$. La molécula es plana y de simetría puntual $\mathrm{D}_{2 \mathrm{~h}}$.

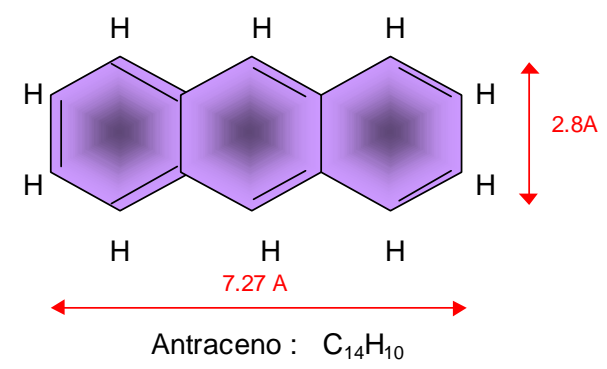

Fig. 1 Molécula de antraceno.
La estructura cristalina de Antraceno [12] es: sistema monoclínico y simetría $\mathrm{C}_{2 \mathrm{~h}}{ }^{5}$. La métrica de la celda unitaria $(300 \mathrm{~K})$ es: $\mathrm{a}=8.56 \AA \AA$ ㅇ $\mathrm{b}=$ $6.04 ; \mathrm{c}=11.18 \AA$.

\section{ANTRACENO}

\section{Diagrama energético}

El modelo energético de Antraceno se muestra en la Fig. 2 y es característico de todos los cristales moleculares [7]: el estado electrónico fundamental $\mathrm{S}_{\mathrm{o}}$ está compuesto por un cierto número de niveles vibrónicos distanciados uno de otro aproximadamente $174 \mathrm{meV}$, valor que corresponde a la vibración molecular predominante de antraceno de $1403 \mathrm{~cm}^{-1}$.

\section{Antraceno: Cristal}

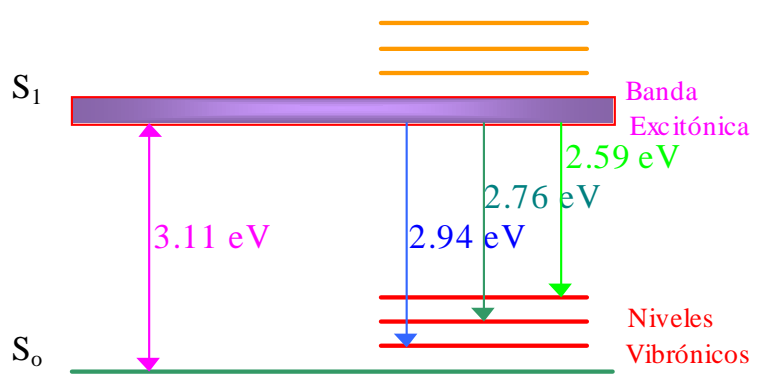

Fig. 2 Representación esquemática de los niveles de energía del cristal molecular antraceno.

El primer nivel electrónico excitado $\mathrm{S}_{1}$ está compuesto a su vez de la Banda Excitónica Frenkel y niveles vibrónicos superiores.

El cristal de antraceno muestra así un cuasigap de $3.11 \mathrm{eV}(398.45 \mathrm{~nm})$.

\section{ANTRACENO}

\section{Parámetros ópticos}

Entre los cristales poliacénicos, el antraceno es uno de los más estudiados [13], limitándonos en esta parte a destacar los valores más significativos.

\section{INDICES DE REFRACCIÓN}

La elipsoide de índices de refracción (Indicatriz) está formada por los ejes: $\mathrm{n}_{\alpha}=1.55 ; \mathrm{n}_{\beta}=1.77 ; \mathrm{n}_{\gamma}=$ 
2.04; el eje $\gamma$ está inclinado el ángulo $\varphi=118.5^{\circ}$ del eje cristalográfico a.

El cristal Antraceno posee 2 ejes ópticos separados por el ángulo $\Omega=44.3^{0}$, centrados alrededor del eje $\gamma$, en el plano perpendicular al eje $\beta[14]$.

La morfología típica de formación de los cristales de antraceno es de forma laminar, clivándose fácilmente en planos paralelos. Las placas planoparalelas de antraceno son a su vez paralelas a los ejes cristalográficos $\mathbf{a}$ y $\mathbf{b}$.

El eje intermedio $\beta$ de la indicatriz coincide así con el eje cristalográfico b,y el eje menor $\alpha$ se encuentra levantado un ángulo de $28.5^{\circ}$ con respecto al eje cristalográfico a y perpendicular al eje cristalográfico b. Los índices de refracción paralelos al plano preferencial son así: $\mathrm{n}_{\beta}=1.77$ y $\mathrm{n}_{\alpha}{ }_{\alpha}=1$ 1.65.Los índices de refracción indicados son referenciales y corresponden a $\lambda=589 \mathrm{~nm}$.

\section{COEFICIENTES DE EXTINCIÓN}

Las medidas del coeficiente de extinción $\kappa$ en láminas de antraceno, dependen de la polarización empleada. En el caso de luz polarizada según el eje b, la absorción de antraceno se debe a dos contribuciones: $\kappa_{1}$ y $\kappa_{2}[15]$.

$$
\kappa_{1}=\kappa_{01} \exp \left[\sigma\left(\mathbf{h} \nu-\mathbf{E}_{0}\right) / \mathbf{k T}\right]
$$

donde: $\mathrm{E}_{0}=3.115 \mathrm{eV}, \sigma=0.88, \kappa_{01}=6 \times 10^{4}$ $\mathrm{cm}^{1}$.

$$
\kappa_{2}^{*}=\kappa_{02} \exp [-\mathrm{E} / \mathrm{kT}]
$$

donde: $\mathrm{E}=0.16 \mathrm{eV}, \kappa_{02}=1.2 \times 10^{5} \mathrm{~cm}^{-1}$.

El coeficiente $\kappa_{1}$ crece con la energía a partir de $E_{0}$ (Ley de Urbach).

El coeficiente $\kappa_{2}$ crece con energías menores que $\mathrm{E}_{0}$ hasta aproximadamente $2.992 \mathrm{eV}$, donde se tiene su máximo $\left(\kappa_{2}{ }^{*}\right)$.

Esta contribución es causada desde niveles vibrónicos, térmicamente excitados, del estado fundamental $\mathrm{S}_{0}$ hasta el primer estado electrónico excitado $S_{1}$

\section{PARTE EXPERIMENTAL}

En esta sección se describen los principales métodos seguidos en la caracterización de las muestras estudiadas, así como los resultados obtenidos.

\section{ELABORACIÓN DE CRISTALES DE ANTRACENO}

El método que utilizamos para obtener cristales de antraceno a partir de una muestra en polvo de ésta sustancia es el método de sublimación.

A continuación (Fig. 3) describiremos el equipo utilizado así como el procedimiento seguido: En el interior de un horno Pirex $(500 \mathrm{~W})$, alimentada por una fuente (Variak), se tiene suspendida la cámara de sublimación.

La Cámara a su vez es un recipiente evacuable Pirex que permite sostener la placa de cristalización. Colocándose el material de base (Antraceno en polvo) en la parte inferior de la cámara. Una vez depositado el antraceno en el interior de la cámara de sublimación se procede a evacuar ésta (Bomba de vacío). La cámara posee un adaptador de acople para la bomba de tal manera que esta quede herméticamente cerrada.

Luego de alcanzar el vacío de trabajo se inyecta nitrógeno, tal que el proceso de sublimación se desarrolle en esa atmósfera.

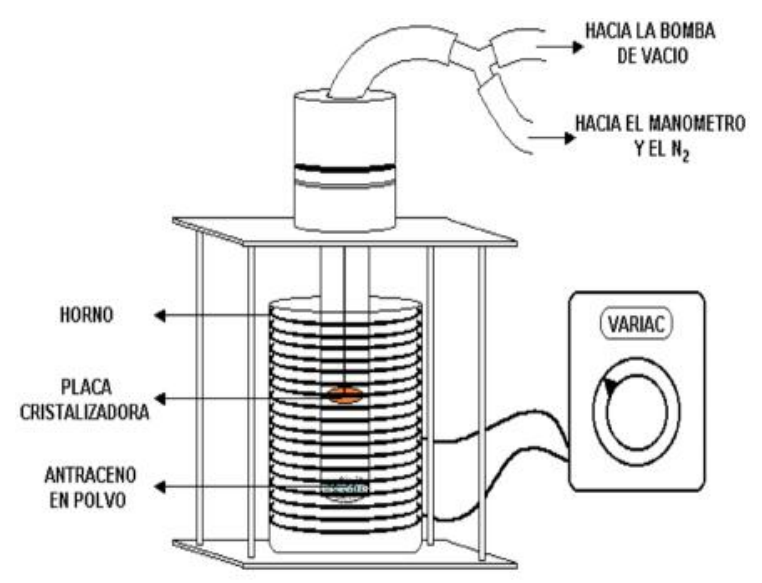

Fig. 3 Sistema de sublimación empleado en la elaboración de cristales de antraceno. 


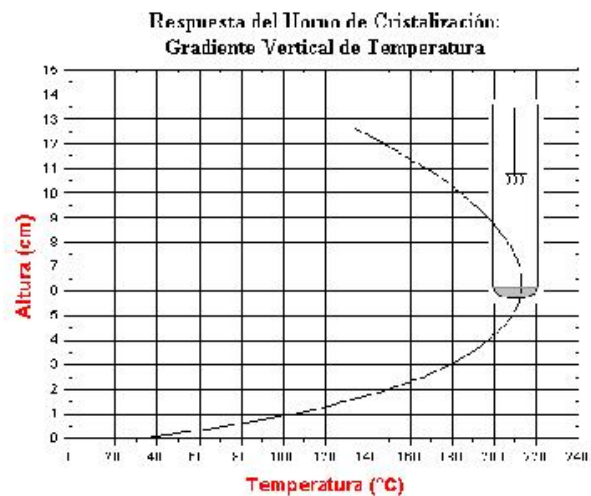

Fig. 4 Detalle técnico del método de sublimación empleado en este trabajo.

En la Fig. 4 se muestra de manera esquemática la configuración de temperatura que debe efectuar el sistema Horno / Cámara de sublimación, tal que se produzca la recristalización de antraceno en la parte superior (Placa de cristalización).El resultado va a depender fuertemente de la temperatura de equilibrio que se alcance en el horno, de la posición relativa de la cámara de sublimación en el horno y del tiempo que dure el proceso. En este trabajo, los mejores resultados se obtuvieron en el lapso de 10 minutos en las condiciones de geometría y temperatura descritos en la Fig. 4.

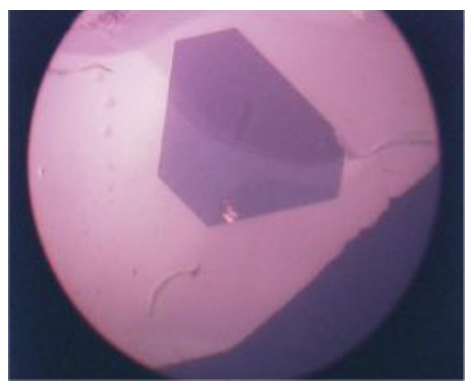

Fig. 5 Cristal de Antraceno observado entre dos polaroides cruzados.

Los Cristales de Antraceno obtenidos en este trabajo se caracterizan por poseer: geometría hexagonal (Fig. 5) con diámetros de $1 \mathrm{~mm}$ a $7 \mathrm{~mm}$ Aprox. Un espesor uniforme (de $0.2 \mu \mathrm{m}$ a $1.5 \mu \mathrm{m}$ Aprox.); lo que se puede apreciar mediante la observación de polaroides cruzados (Fig. 5): color definido en toda su superficie.

Las caras plano paralelas de los cristales obtenidos contienen los ejes cristalográficos a y b, coincidiendo siempre dos cantos del hexágono formado con el eje b, como se observa en la Fig.6.

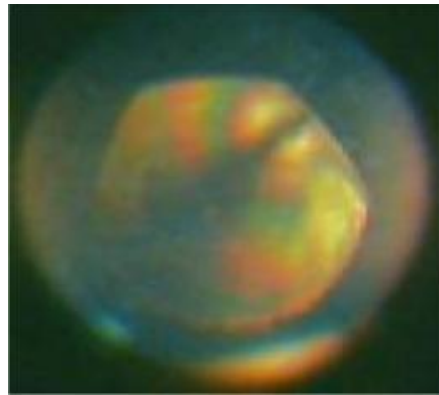

Fig. 6 Cristal de Antraceno en observación conoscópica.

En la Fig. 6 se muestra la observación conoscópica de un cristalito típico de Antraceno, de geometría hexagonal. Se distingue la estela isogira (color negro) que se origina en un vórtice superior (eje óptico) y se va ensanchando hacia la parte inferior. La línea central que divide la estela isogira de modo simétrico, coincide con el eje cristalográfico a, que como se aprecia va a cortar perpendicularmente a uno de los cantos del cristal, el cual coincide con el eje cristalográfico b.

\section{MEDICIÓN DE TRANSMITANCIA}

En la Fig. 7 se muestra esquemáticamente el arreglo experimental empleado en la medición de transmitancia Frontal de Antraceno. Para efectos de montaje el cristal se adhiere a una lámina de vidrio y es sometida a la irradiancia de una lámpara blanca (Lámpara de Deuterio, $50 \mathrm{~W}$ ). La señal transmitida es enfocada a la entrada de un analizador Multicanal óptico (OMA), el cual procesa la señal y presenta finalmente el espectro de transmisión de la muestra.

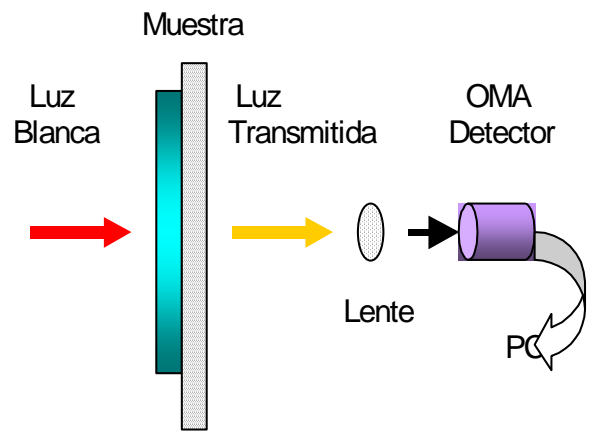

Fig. 7 Medición de transmitancia.

El resultado de la medición se muestra en la Fig.8. 
De este espectro se puede observar la fuerte opacidad de Antraceno para longitudes de onda menores a 398 nm (ó $3.12 \mathrm{eV}$ ).

El valor encontrado coincide muy bien con el valor referencial del cuasigap de Antraceno (3.115 eV).

No obstante debido a los efectos de polarización en futuras medidas de este tipo se debe incluir un analizador de polarización.

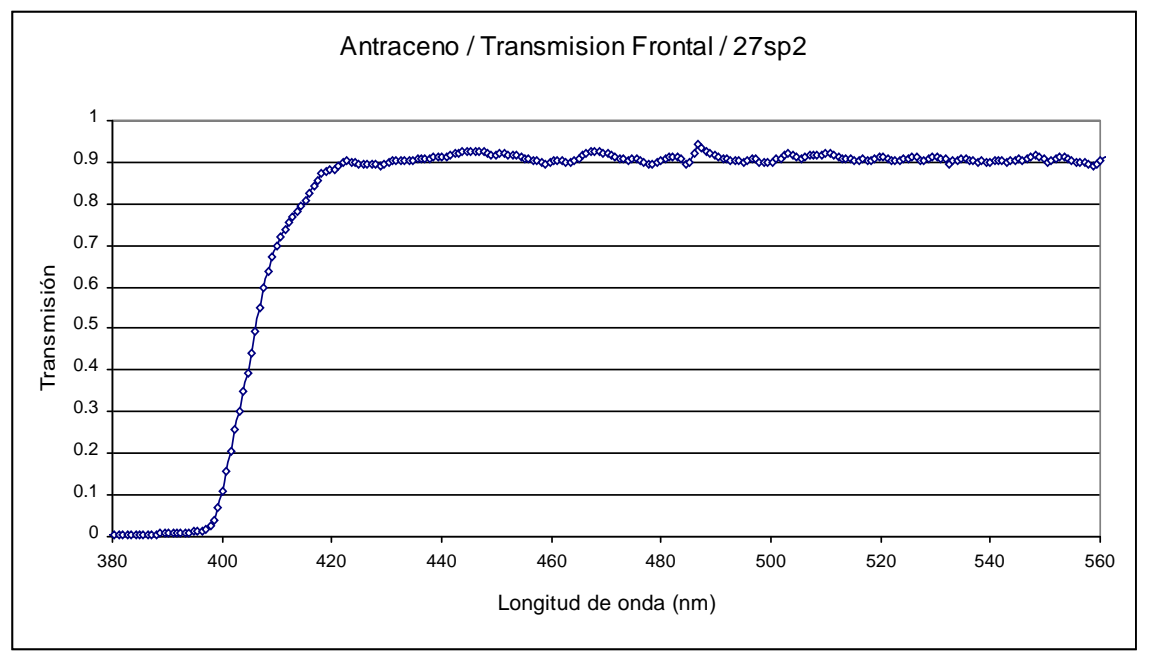

Fig. 8 Espectro de transmisión frontal no polarizado de un cristal de antraceno.

\section{MEDICIÓN DE FLUORESCENCIA}

A diferencia de las medidas de transmisión descritas anteriormente, que se realizaron en un arreglo Frontal, las medidas de fluorescencia que a continuación se describen, se realizan en un arreglo transversal (Fig. 9).La incidencia frontal de radiación UV (Lámpara de deuterio) produce la fluorescencia localizada de antraceno, la misma que se propaga vía Modos de guía de onda (Wave guide emission, Fig. 10) hacia los bordes del cristal, donde el detector D de fibra óptica los capta y los deriva al Sistema OMA,Fig. 11.

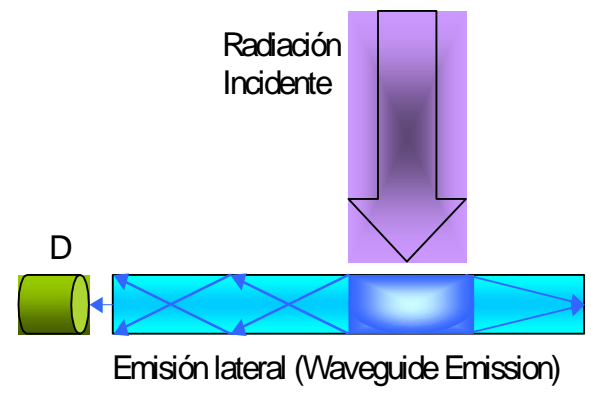

Fig. 9 Excitación transversal.

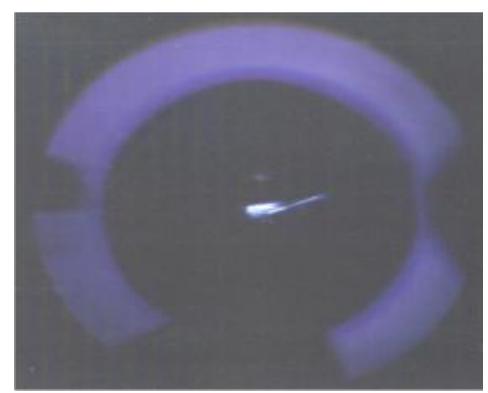

Fig. 10 Cristal de antraceno, fluorescencia lateral (wave guide emisión).

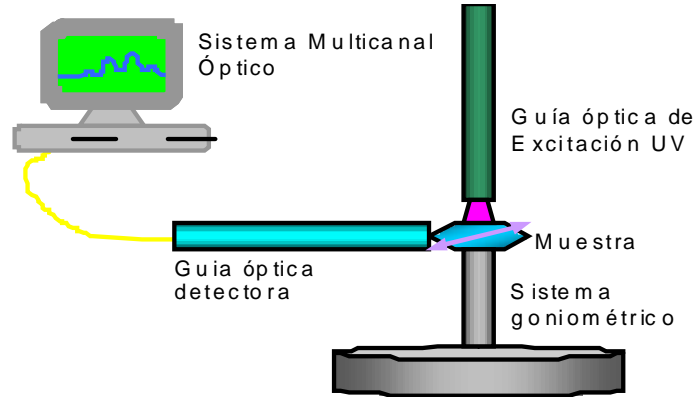

Fig. 11 Sistema giroscópico empleado en la excitación y análisis de la fluorescencia de cristales de Antraceno. 


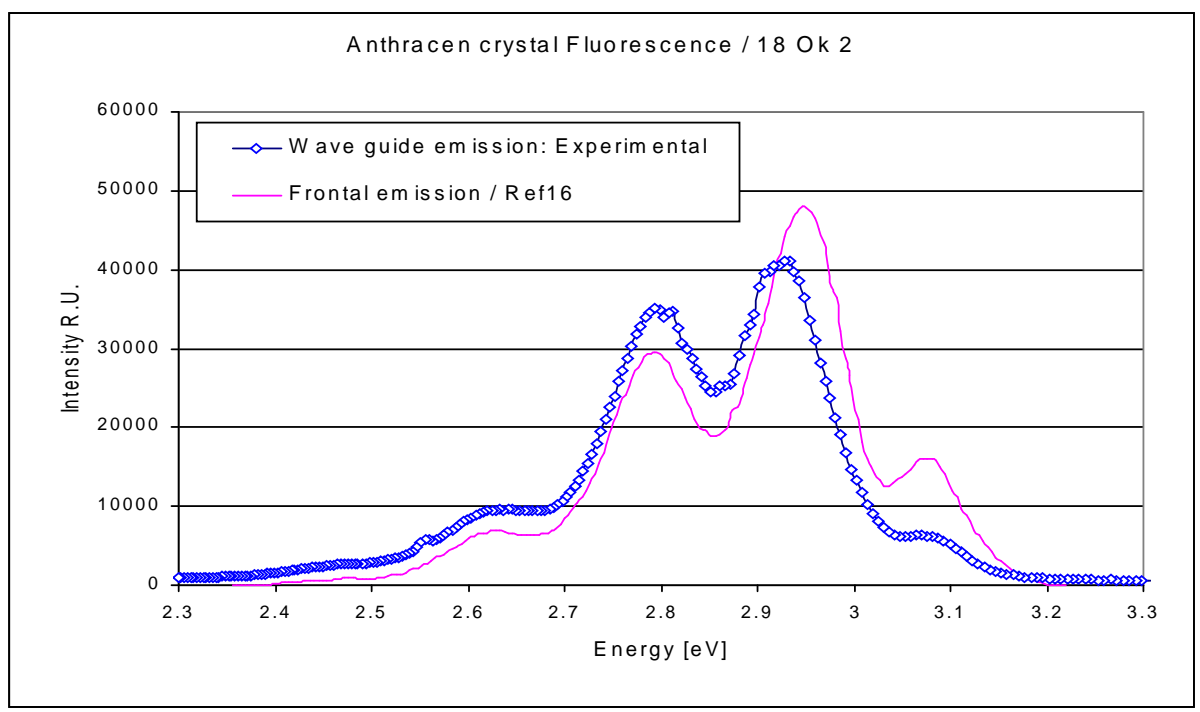

Fig. 12

Espectro de Fluorescencia de canto (Wave guide emisión) de un cristal de Antraceno (UNI) y espectro de fluorescencia frontal referencial [16].

En la Fig. 12 se presenta el resultado del análisis espectral de la fluorescencia de canto emitida por un cristal de antraceno en este trabajo. A modo de comparación se acompaña el espectro de fluorescencia frontal tomado de la referencia 16.

La respuesta experimental permite apreciar la ocurrencia de 4 máximos relativos, localizados en: $3.08,2.92,2.79$ y $2.61 \mathrm{eV}$.

De otro lado, la curva referencial evidencia también 4 máximos relativos en las posiciones: $3.07,2.94,2.79$ y $2.61 \mathrm{eV}$. Es de resaltar la notable coincidencia espectral, no obstante esperarse mayor diferencia, debido al proceso de reabsorción, la que debería ser significativamente mayor en el caso de la Fluorescencia de canto, tal como se evidencia en la disminución de intensidad relativa de las bandas más energéticas.

El resultado obtenido se explica en el marco del diagrama energético de Antraceno presentado en la Fig.2, en donde se detalla las principales transiciones energéticas: $3.11,2.94,2.76$ y 2.59 $\mathrm{eV}$, correspondiendo estas, respectivamente a las transiciones: $\mathrm{S}_{1} \rightarrow \mathrm{S}_{0}, \mathrm{~S}_{1} \rightarrow\left(\mathrm{S}_{0}+\mathrm{v}_{0}\right), \mathrm{S}_{1} \rightarrow\left(\mathrm{S}_{0}+\right.$ $\left.2 \mathrm{v}_{0}\right), \mathrm{S}_{1} \rightarrow\left(\mathrm{S}_{0}+3 \mathrm{v}_{0}\right)$, donde $\mathrm{v}_{0}$ es el nivel de energía vibrónico $1,403 \mathrm{~cm}^{-1}$ (ó $174 \mathrm{meV}$ ).

Las leves diferencias de correspondencia se explican por efectos de temperatura y el efecto de reabsorción.

\section{MEDICIÓNES DE FOTOCORRIENTE}

En base a monocristales puros de Antraceno se realizan uniones fotovoltaicas, midiéndose su eficiencia cuántica espectral.

\section{Espectrómetro de fotocorriente}

En la Fig. 13 se muestra esquemáticamente el espectrómetro de fotocorriente empleado. El Sistema es un montaje de Laboratorio, en el cual una fuente de radiación Lamp (Lámpara Halógena, $650 \mathrm{~W}$ ) es modulada por un Chopper y dirigida hacia un Monocromador (300 nm - 2500 $\mathrm{nm})$. La radiación monocromática es dirigida finalmente sobre la muestra fotovoltaica Probe. La respuesta de fotocorriente es detectada por un amplificador Lock-In y derivada a una PC para su almacenamiento y procesamiento.

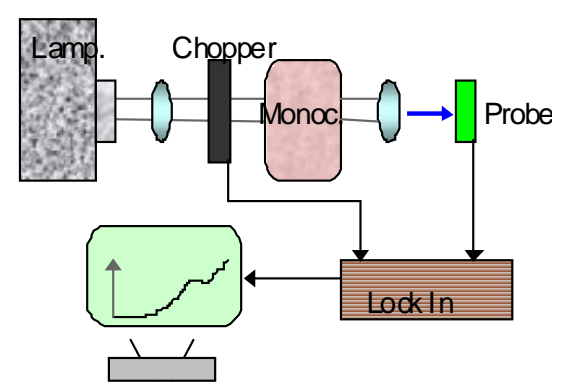

Fig.13 Espectrómetro de fotocorriente. 


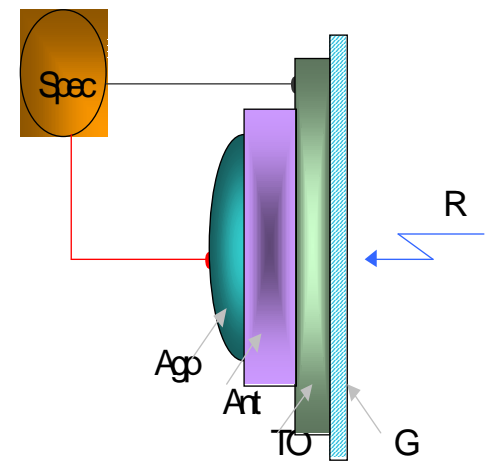

Fig. 14 Muestra fotovoltaica en base a un monocristal de Antraceno.

En la Fig. 14 se describe esquemáticamente la configuración fotovoltaica construida: Sobre una lamina de vidrio $\mathbf{G}$ se deposito previamente por el método de Spray pirolisis una capa de oxido de estaño TO (Tin Oxide), que actúa como contacto transparente. Sobre el substrato de TO se adhirió una película cristalina de antraceno ANT $(\sim 1 \mu \mathrm{m}$ de espesor), y finalmente sobre la película de antraceno se adhirió pasta de plata Agp para establecer el contra contacto eléctrico.

El espectro muestra un fondo estructurado con 2 contribuciones muy débiles $(1.28 \mathrm{eV}$ y $1.9 \mathrm{eV})$.

A partir de 2.78 eV la contribución de fotocorriente es relativamente importante, dejándose expresar por:

$$
\eta_{3}=[\exp (-14.13)] *[h v-2.78]
$$

donde: $h v$ es la energía fotónica incidente.

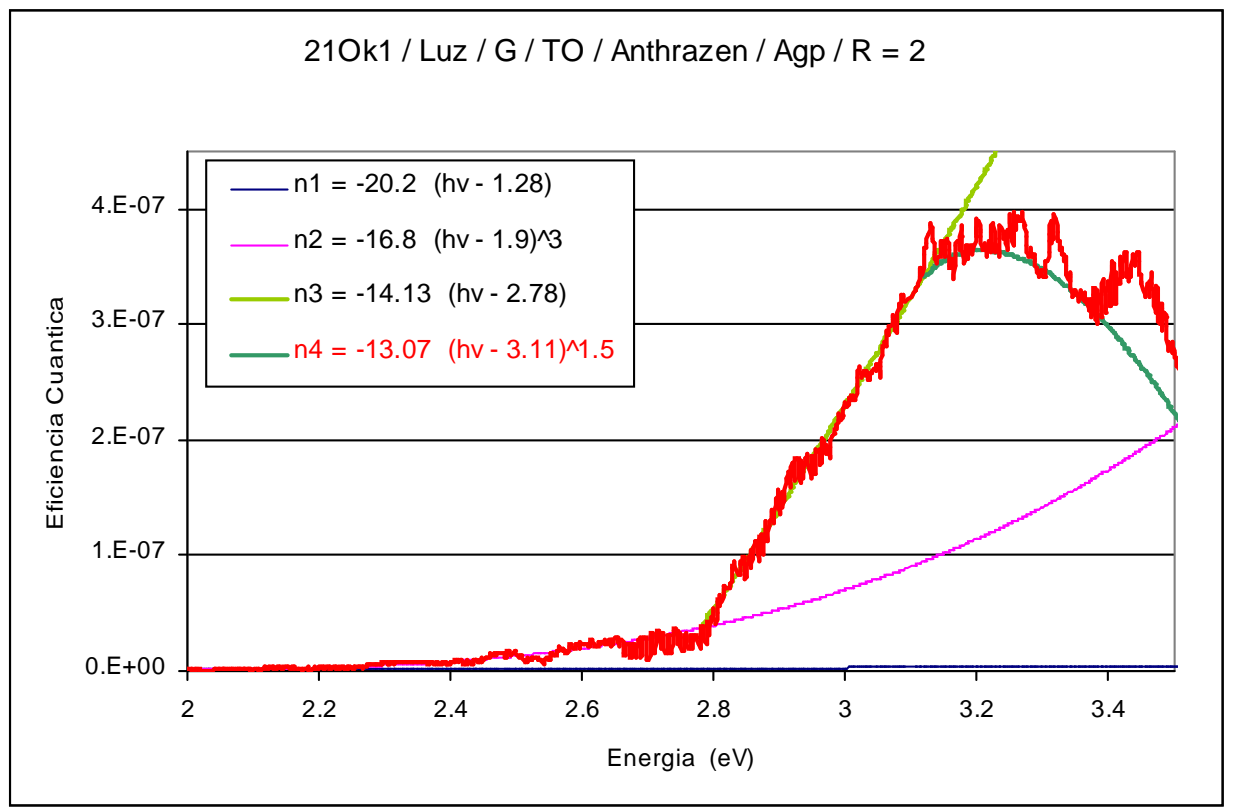

Fig. 15 Espectro de eficiencia cuántica de la unión fotovoltaica en base a antraceno descrito en el esquema 14.

En la Fig. 15 se muestra uno de los espectros de eficiencia cuántica obtenidos.

La respuesta fotovoltaica mostrada a partir de 2.78 $\mathrm{eV}$ es frenada a partir de $3.11 \mathrm{eV}$, lo cual es descrito por la contribución negativa $\eta_{4}$ :

$\eta_{4}=-[\exp (-13.07)]^{*}[\mathrm{~h} v-3.11]^{1.5}$

\section{Modelo energético de la unión fotovoltaica}

En la Fig. 16 presentamos el modelo energético más probable de la unión fotovoltaica construida. En este modelo, la fotogeneración detectada a partir de $2.78 \mathrm{eV}$ la identificamos como el proceso 1, ocurrente dentro de antraceno cerca al contacto con plata (Agp), por el cual, son generados electrones a la banda $S_{1}$ desde un nivel $2.78 \mathrm{eV}$ por 
debajo de la banda (ó $0.33 \mathrm{eV}$ por arriba del nivel fundamental $\mathrm{S}_{0}$ ).

El decrecimiento gradual de la señal fotovoltaica a partir de $3.11 \mathrm{eV}$ (Cuasigap de Antraceno), se explica de un lado por el amortiguamiento de la radiación (R) que llega a la zona 1 (Proceso de absorción) más una leve generación de fotoportadores en la zona de entrada (Proceso 2, Fig. 16).

Las contribuciones menores (1.28 y $1.9 \mathrm{eV})$ se deben probablemente a transiciones desde estados superficiales del material TO y que son motivo de un análisis posterior.La justificación de las aserciones efectuadas en este modelo se hará en la sección correspondiente a la discusión de los resultados.

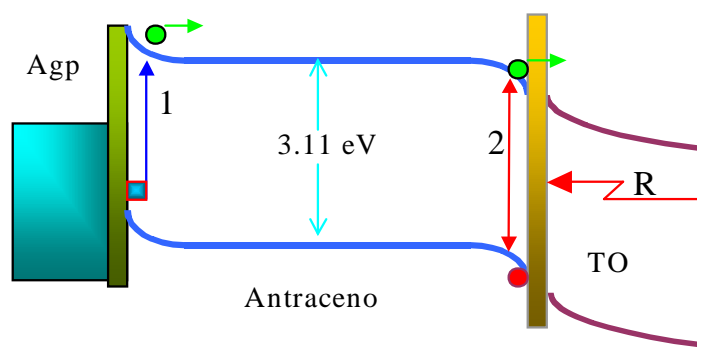

Fig. 16 Modelo energético de la unión fotovoltaica construida en este trabajo mediante un monocristal de antraceno.

\section{DISCUSIÓN DE RESULTADOS}

Como es de observar, la metodología seguida en este trabajo conlleva a la realización de un conjunto de mediciones complementarias, que permiten definir gradualmente el material elaborado y sus propiedades, teniendo como base de comparación ciertos datos referenciales. Este procedimiento es en nuestro caso, de mayor importancia, debido a las diferencias evidentes entre las condiciones de trabajo locales y los estándares internacionales (por ejemplo: materiales de base de alta pureza, equipamiento especializado).

En lo referente a los resultados de observaciones de morfología y calidad cristalográfica de las muestras elaboradas, se ha demostrado que estos son comparables a los obtenidos en Laboratorios de gran experiencia [13].

La medición de la transparencia frontal de Antraceno, permite estimar el cuasigap del material $(3.11 \mathrm{eV})$, no obstante, la medida es incompleta, ya que no se incorporo un analizador de radiación, ni se separo la radiación Visible proveniente directamente de la lámpara de la fluorescencia frontal proveniente del propio antraceno.

Las mediciones de Fluorescencia de canto (Wave guide emisión) se ajustan muy bien a lo esperado y constituye la base de un próximo estudio a publicar (Emisión Láser de cristales de Antraceno a temperatura ambiente).

El resultado obtenido en la configuración fotovoltaica en base a Antraceno merece mayor explicación. Ante todo debemos mencionar que si bien la señal fotovoltaica obtenida es muy baja y se encuentra en el límite de detección de nuestro equipo, toda respuesta obtenible constituye información valiosa del material (Antraceno) y sus uniones.

De las diversas alternativas de configuración fotovoltaica del tipo (Metal / antraceno / TO), intentada hasta el momento en nuestro Laboratorio, solo en el caso Metal = pasta de plata (Agp), hemos obtenido una respuesta medible. Esta es otra de las razones, además del amortiguamiento posterior, por la que consideramos que la fotogeneración de $2.78 \mathrm{eV}$ tiene lugar cerca de la interfase Agp/Antraceno.

El semiconductor TO elaborado en nuestro laboratorio (Spray pirolisis) muestra efecto fotovoltaico en uniones directas del tipo (Metal / TO), sin embargo en ningún caso hemos detectado la generación de una señal apreciable en el rango 2.7 a $3.5 \mathrm{eV}$, por lo que consideramos que la respuesta reportada en este trabajo en ese rango se debe a la interacción del Antraceno.

El origen de la transición correspondiente a 2.78 $\mathrm{eV}$ lo hemos situado a $0.33 \mathrm{eV}$ por arriba del nivel fundamental de Antraceno por coincidir con uno de los niveles vibrónicos mas fuertes de Antraceno $(2.79 \mathrm{eV})$, lo cual aún no es definitivo, ya que podría ser que la pasta de plata produzca estados superficiales o Traps adicionales en el Antraceno. 
Los campos eléctricos de las uniones de Antraceno con pasta de plata (Agp) y respectivamente con oxido de estaño (TO) los hemos dispuesto del mismo signo, debido al hecho experimental, que en caso contrario los efectos son mucho más drásticos (por ejemplo: cambio de signo en la foto respuesta).

El hecho de haber obtenido en la respuesta fotovoltaica 2 energías características de antraceno $(2.78$ y $3.11 \mathrm{eV})$ es un indicador positivo de la certeza de tener respuesta fotovoltaica del material antraceno.

\section{CONCLUSIONES}

El trabajo aquí descrito tiene como contribución importante, presentar las técnicas experimentales más apropiadas, para la elaboración y caracterización de cristales orgánicos, aplicando al caso particular de antraceno.

Las que constituyen la base de próximos trabajos y aplicaciones a efectuar en nuestro Laboratorio con este tipo de materiales.

Como contribución secundaria, se debe mencionar la realización de una celda fotovoltaica en base a un cristal de Antraceno y la comprobación de efectividad del método de fotocorriente espectral en el estudio de los semiconductores orgánicos.

\section{RECONOCIMIENTO}

Este trabajo se realizó gracias al apoyo económico de la UNI / RECTORADO, que financio la adquisición de un Equipo Analizador Multicanal Óptico.

Las mediciones de fotocorriente espectral se realizaron mediante un equipo construido $\mathrm{y}$ automatizado en el Laboratorio por el Prof. Guido Castillo.

Nuestro reconocimiento a la Prof. Susana Petrick por su colaboración con los trabajos de microscopía y tomas fotográficas.

Nuestro mayor agradecimiento al Sr. Prof. Dr. Norbert Karl de la Universidad de Stuttgart / Alemania, por las enseñanzas recibidas y la motivación de este trabajo.

\section{REFERENCIAS}

1. Tang, C. W., "Appl. Phys. Lett”. 48: pp.183, 1986.

2. Forrest, S. R., F. F. So., “J. Appl. Phys". Vol. 64, pp. 399, 1988.

3. Dodabalapur, A., Rothberg, L. J., Miller, T. M., “Appl. Phys”. Lett. 65, pp. 2308. 1994.

4. Dodabalapur, A., Torsi. L., Katz, H. E., "Science", pp. 268, 270. 1995.

5. Zang, D. Y., S. R. Forrest. "IEEE Photon. Technol. Lett". V. 4, pp. 365. 1992.

6. So, F. F., Forrest. S. R., "IEEE Trans. Electron. Dev". Vol. 36, pp. 66. 1989.

7. Valera, A., "Laseremission aus Frenkelexcitonenzustaenden von reinem Anthrazen, und 2, 3-Dimethylanthrazen". Doktorarbeit, Universitaet Stuttgart, 1979.

8. Kepler, R. G., “Phys. Rev'.pp.119, 1226, (1960).

9. LeBlanc, O. H., “J. Chem. Phys”. pp.33, 626. (1960)

10.Karl, N., Marktanner, J. Mol., "Cryst. Liq. Cryst". Pp.355, 149. 2001.

11.Rostalski, J., Meissner, D. Solar., "Energy Mater. Solar Cells 63". 2000. pp. 37-47.

12.Mason, R., "Acta Cryst". pp.17, 547. 1964.

13.Glockner, E., Doktorarbeit, Universitaet Stuttgart, III Phys. Inst. 1973.

14.Nakada, I., “J. Phys”. Soc. Japan, 19, 11962.

15.Nakada, I., "J. Phys". Soc. Japan, 19, 346 1965.

16.Logan, L. M., Munro, I. H., Williams, D. F., Lipsett, F. R., from "Molecular Luminescence", Ed. Benjamin, pp. 773-785, N.Y. 1969.

Correspondencia: mmosqueram@uni.edu.pe 